\title{
Influencia de la robótica educativa en la motivación y el trabajo cooperativo en Educación Primaria: un estudio de caso
}

\author{
Influence of Educational Robotics on Motivation and Cooperative \\ Learning in Primary Education: a Case Study
}

iD Tania Sánchez Sánchez

CEIP San José Artesano, Abarán, Murcia, España

taniassanche@gmail.com

(iD) José Luis Serrano Sánchez

Departamento de Didáctica y Organización Escolar, Universidad de Murcia, España

jl.serranosanchez@um.es

D Fulgencio Rojo Acosta

CEIP Juan Navarro, La Hoya de Lorca, Murcia, España

fulgencio.rojo@murciaeduca.es

\section{RESUMEN}

En este artículo analizamos la influencia que la robótica educativa tiene en la motivación de los estudiantes de Educación Primaria en un Colegio de la Región de Murcia (España), así como el impacto que tiene en el trabajo cooperativo. En este estudio de caso han participado 18 estudiantes y su docente. El cuestionario y la entrevista han sido las técnicas utilizadas para la recogida de información. Hemos podido comprobar que las actividades en las que se ha integrado la robótica educativa han tenido una influencia positiva en la motivación del alumnado, en su interés y en la mejora de la consolidación de aprendizajes.

PALABRAS CLAVE robótica, motivación, aprendizaje cooperativo, educación primaria.

\section{ABSTRACT}

In this article we analyze the influence that educational robotics has on the motivation of Primary School students in a School in the Region of Murcia (Spain), as well as the impact it has on cooperative learning. In this case study 18 students and their teacher participated. The questionnaire and the interview have been the techniques used for the collection of information, with which we have been able to verify that the activities in which the educational robotics have been integrated have had a positive influence on the motivation of the students, in their interest and in the improvement of learning consolidation.

KEYWORDS robotics, motivation, cooperative learning, primary education. 


\section{INTRODUCCIÓN}

En este estudio se analizan las posibles relaciones que hay entre la robótica educativa (RE) y los factores que influyen en la motivación del alumnado durante un proyecto robótico, así como su relación con el trabajo cooperativo. La investigación se ha llevado a cabo en un colegio de Lorca de la Región de Murcia (España). Participan en esta experiencia tanto el alumnado de un aula de $6^{\circ}$ de Educación Primaria como su docente, que a su vez es el coordinador del proyecto de RE del centro.

\subsection{La robótica educativa en Educación Primaria}

La RE permite crear "un entorno que puede capacitar a un docente para estimular a los estudiantes a reflexionar sobre sus propias ideas, alentándolos a comparar estas ideas con el conocimiento científico conocido y aceptado, y buscar establecer el vínculo entre estos dos tipos de conocimiento" (Jófili, 2002, citado en Vigueras y Villalba, 2017, p.3). A partir de ahí nos encontramos, como explica Ruiz (2007), una generación con la que se trata de desarrollar unos ambientes de aprendizaje basados en la tecnología. El fin último es provocar aprendizajes significativos en los estudiantes, de manera interdisciplinar y holística, para tratar de desarrollar habilidades de resolución de problemas en equipo.

Barrera (2015), destaca el entusiasmo que provoca en los estudiantes la construcción de saberes, favoreciendo el uso responsable y crítico de la tecnología. La robótica se puede convertir, por lo tanto, en un medio para tratar de comprender, crear y volver a aprender la realidad en la que se vive. Cabrera (2015), por su parte, subraya el desarrollo de habilidades transversales que se trabajan mediante el uso de la programación, mientras que García y Reyes (2012) destacan las diversas investigaciones que han demostrado que la RE tiene un efecto positivo en la motivación en el aula.

Con la RE podemos permitir que los estudiantes activen su motivación a partir del estímulo de la curiosidad científica, la indagación y experimentación (Barrera, 2015), permitiéndoles descubrir un medio natural donde "a través del juego puede interactuar y desempeñar un rol dentro de situaciones didácticas provenientes de la realidad; con las cuales ellos deben generar estrategias para la planificación, ejecución y solución de una situación problema" (Acosta et al., 2015, p. 18), mediante el diseño, programación y manipulación del robot.

Para poder llevar a cabo la RE en el aula nos encontramos con una variedad de recursos que pueden ser adaptados a las necesidades de cada grupo de estudiantes. Nos encontramos con múltiples propuestas didácticas y eventos que, en no pocas ocasiones, son promovidos por empresas. De esta manera, además de proporcionar robots adaptados, tratan de cubrir los déficits de formación que tienen los propios docentes.

Diversas son las iniciativas educativas con el uso de robots. González y Redondo (2013) han trabajado en el proyecto AlToy con el fin de producir un juguete con fines educativos a través del uso del robot AlSoy, un robot emocional que se adapta a la competencia lingüística, social y comunicativa. Por su lado, Cubides et al. (2012) han realizado un proyecto basado en el diseño, la construcción y la implementación de una plataforma robótica multifuncional con usos didácticos, denominada "Dingo 1.0". Cervera y Casañ (2015) analizan la red RPN, una iniciativa con la finalidad de crear una "red de laboratorios educativos de robótica con capacidades de programación remota. Consiste en materiales abiertos en línea y servidores que están preparados para que los estudiantes puedan probar sus programas en el mismo momento que los realizan" (p. 63). 
En España, desde 2006, nos encontramos que en diversas Comunidades Autónomas se están realizando la conocida como First Lego League (FLL), una competición sobre RE y dirigida a alumnos entre 10 y 16 años. En esta competición se participa en grupos donde se plantea una problemática del mundo real que, cada grupo, deberá resolver a través de la creación de un robot utilizando la tecnología Lego Mindstorms. También nos encontramos con una FLL Junior, dirigida a alumnos de entre 6 y 9 años, que se desarrolla utilizando elementos de LEGO Education WeDo 2.0. Como tercera modalidad, encontramos la FLL Tech Challenge, dirigida a jóvenes de entre 16 y 20 años, donde los participantes realizan diseños, creaciones, pruebas y programan robots autónomos que se controlan de forma remota.

La RE puede ser, por tanto, un recurso educativo que no busca el fin de un aprendizaje, sino que se trata de un medio para llegar a este. Como afirma Barrera (2015) o García y Reyes (2012), no se pretende que todos los estudiantes lleguen a ser programadores profesionales sino que, a través de los beneficios que aporta la RE en el aula, el alumnado llegue a adquirir conocimientos más complejos y significativos, despertando así el interés y la motivación por su propio proceso de enseñanza-aprendizaje a la par que mejoran sus habilidades de resolución de problemas en equipo.

\subsection{Robótica educativa y motivación}

Podemos definir la motivación como "los mecanismos de activación con acceso relativamente directo a las vías motoras, que tienen el potencial de facilitar y dirigir ciertos circuitos motores mientras inhiben otros", es decir, "los mecanismos que nos hacen desear y decidir hacer ciertas cosas" (Jiménez, 2017, p. 23). La toma de decisiones se realiza a través de un proceso neuronal que está en funcionamiento a cada momento, por lo que se considera que todo lo que se ha hecho o dejado de hacer es fruto de esta toma de decisiones, a veces de forma más automática y otras más reflexionadas. La acción de decidir y el deseo, junto con la respuesta placentera o hedónica que sentimos al realizar una acción que nos motiva y que refuerza las conexiones neuronales - facilitando la repetición futura de esta acción-, dan lugar a un proceso cerrado que se va retroalimentando a lo largo del tiempo.

FIGURA 1. Ciclo de la motivación final (Jiménez, 2017)

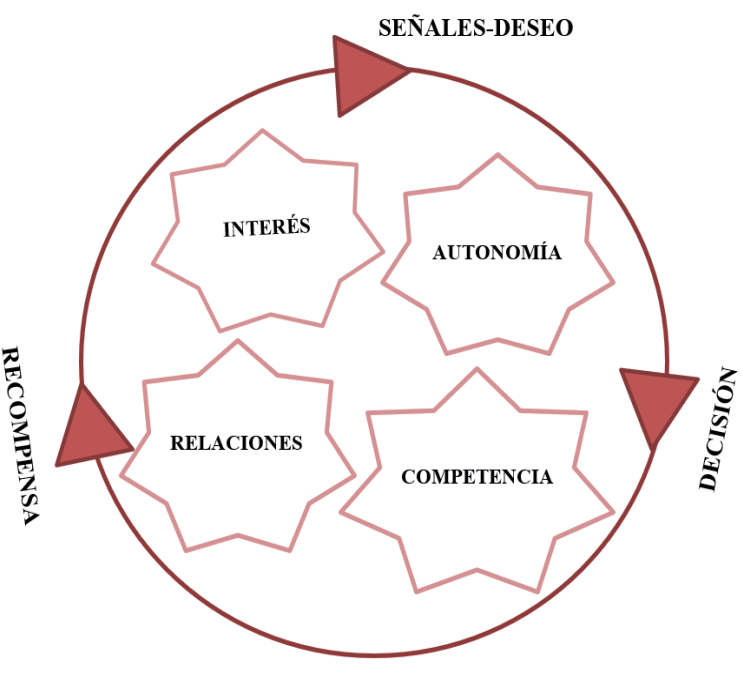

La motivación es un proceso cognitivo que ha sido estudiado durante muchos años. Jiménez (2017) realiza un riguroso estudio en el que analiza las investigaciones realizadas sobre este mecanismo. El autor define el ciclo de la motivación mediante la integración de dos de las teorías más utilizadas para explicar este concepto: la teoría de la autodeterminación (competencia, autonomía y relaciones) y la de los 16 deseos básicos.

El autor concluye recordando que este ciclo de la motivación no es la receta mágica o infalible para despertar dicho interés en cada persona, ya que aún no se conoce el motivo exacto por el cual una persona, en un momento determinado, desea y decide hacer algo en concreto, o no hacerlo. 
Llevando dicho ciclo a nuestro contexto, y tal y como afirman Moreno et al. (2012 p. 79), "los robots son utilizados en el aula como herramienta que favorece el acercamiento de un modo diferente a los contenidos del currículo, y que por sus propias características facilitan el aprendizaje por indagación”. Autores como Cabero et al. (2017) y Merino et al. (2017) destacan que, en los estudiantes, "la motivación que despierta el uso de robots programables para la resolución de las actividades planteadas se puede considerar positiva" (p.171). En la misma línea encontramos a García y Reyes (2012), que exponen que existen diversas investigaciones, donde destacan el efecto positivo de la motivación en el aula a partir del uso de la robótica, así como la investigación de Fagin y Merkle, (2003) que enfatiza el potencial de la RE para resaltar de forma positiva el aprendizaje gracias a la mejora de la motivación de los estudiantes.

García y Reyes (2012) resaltan que la RE tiene, además, una relación directa con el aprendizaje cooperativo, debido a su conexión y beneficios que atribuyen al trabajar con esta metodología durante el desarrollo de un proyecto de RE.

\subsection{Robótica educativa y el trabajo cooperativo}

El mundo actual nos plantea nuevos retos diferentes a los acostumbrados hasta el momento, precisando metodologías y estrategias educativas diferentes para cubrir estas necesidades. Nosotros nos vamos a centrar en este estudio en el trabajo cooperativo en el aula de Educación Primaria, uno de los recursos principales en la mayoría de las metodologías activas.

Una metodología activa se define por estar centrada en el alumno, es decir, convertir a este en responsable de su aprendizaje, necesitando para ello su implicación total y su compromiso para lograr aprendizajes significativos y una conexión entre los diferentes contextos y situaciones en los que se trabaje (Fernández, 2006). Esta misma perspectiva defiende Ayuso (2016), al afirmar que la "responsabilidad del aprendizaje depende directamente del estudiante, generando un aprendizaje más profundo, significativo y duradero facilitando la transferencia a contextos más heterogéneos” (p. 23). Para trabajar estas diferentes interacciones se pueden utilizar técnicas de cooperación que analicen, adapten e integren nuevas técnicas, donde deben estar presentes la importancia de la comunicación y el intercambio de formación a través de la colaboración, la coordinación y la resolución de conflictos (Acosta et al., 2015).

Según Johnson et al. (2004), en el aprendizaje cooperativo se trabaja en pequeños grupos donde los estudiantes se ayudan y logran potenciar el aprendizaje propio y el de los demás. Por su parte, Trujillo (1998) destaca la importancia de la cooperación frente a la competitividad, pues “la ayuda, el compartir, el colaborar y el cooperar se expresan como norma permanente, propiciada en el quehacer de los proyectos como una interdependencia positiva, que cambia la competitividad por la construcción de buenas relaciones entre los diferentes miembros del equipo" (p. 2), favoreciendo así tanto al desarrollo cognitivo individual como al grupal.

En este contexto, el docente posee un rol de mediador y guía en el proceso de aprendizaje de los alumnos, pues facilita las herramientas que necesitan y crea situaciones de aprendizaje con la finalidad de desarrollar en los alumnos una autonomía y las competencias necesarias para hacer frente a una vida completa y de calidad en la sociedad en la que se encuentren (Heredero y Oliva, 2014).

Otro aspecto importante, que destacan Heredero y Oliva (2014), es la organización del aula, pues el agrupamiento heterogéneo es imprescindible para crear ambientes de aprendizaje cooperativo y entre 
iguales, ya que los niños obtienen beneficios de este proceso al enriquecerse personalmente, aprender a aceptar, tolerar y entender mejor a sus iguales.

Dado el carácter interdisciplinar y polivalente de la RE (Acosta et al., 2015; García y Reyes, 2012; Ruiz, 2007; Sánchez et al., 2005), esta puede ser de ayuda para el "desarrollo e implementación de una cultura tecnológica, permitiendo el entendimiento, la mejora y el desarrollo de tecnologías propias a partir de proyectos prácticos y de colaboración para aprender a aprender a partir de la construcción y control de distintos prototipos robóticos con fines didácticos” (p.17). Por su parte, Ruiz (2007) nos indica que nos encontramos en una generación tecnológica donde la RE hace posible el trabajo en unos ambientes de aprendizajes tecnológicos que construyen un cambio significativo en el modelo educativo. En este, el alumno se convierte en un agente activo y participativo en su proceso de aprendizaje, desarrollando habilidades como el respeto, la resolución de problemas y el trabajo en equipo y de forma cooperativa, a través de una visión holística de este proceso.

Morales (2017) afirma que "la RE ha ayudado a los niños a trabajar más y mejor en equipo, escuchar los puntos de vista de los demás, a llegar a una solución entre todas las personas porque saben la importancia de trabajar en equipo" (p. 7). De esta manera, se mejora la capacidad de relación con los demás, a trabajar con otros, a ponernos de acuerdo, etc.

\section{MÉTODO}

\subsection{Objetivos}

La finalidad de esta investigación es analizar el impacto que tiene la RE en la motivación y el trabajo cooperativo entre estudiantes de Educación Primaria de un centro educativo de la Región de Murcia. En este estudio de caso, de tipo descriptivo, se ha seguido un enfoque de investigación mixto, utilizando procedimientos de recogida de información cuantitativos y cualitativos, lo cual nos permite investigar desde una perspectiva más amplia y compleja (Hernández et al., 2010). La elección de este enfoque viene determinada por la necesidad de conocer e indagar sobre el motivo de los resultados obtenidos, puesto que una investigación cuantitativa no nos aporta toda la información necesaria para comprender este objeto de estudio. Por esta cuestión, la acompañamos de un enfoque cualitativo que realizamos a una persona diferente, pero con relación directa con dicho estudio. Esto nos permite entender más los datos, así como poder triangular la información para contrastar o verificar las diferentes ideas obtenidas (Vallejo y De Franco, 2009).

Mediante este diseño, se pretende buscar una descripción o indagación del tema a partir de la obtención de unos datos, los cuales nos harán comprender e interpretar la realidad que encontramos en el aula analizada (Latorre et al., (2005). De esta manera, el estudio de caso nos permite investigar "la particularidad y la complejidad de un caso singular, para llegar a comprender su actividad en circunstancias importantes" (Stake, 2005, p. 11).

El investigador asume un papel participativo durante la parte cualitativa al tener un trato directo con el entrevistado, mientras que en la parte cuantitativa, el rol es de observador, no participando directamente en el desarrollo del proyecto ni en la realización de los cuestionarios, pero sí manteniendo una relación directa con el docente que imparte dicho proyecto y durante su entrevista. 


\subsection{Contexto y participantes}

Esta investigación se ha llevado a cabo en un colegio de Lorca (Murcia). Participaron 18 niños de edades entre 10 y 12 años ( $6^{\circ}$ de Primaria), de los cuales 11 son niñas y 7 son niños. Además, ha participado el docente de estos estudiantes, especialista en Educación Física y coordinador del proyecto de RE del colegio.

En este centro educativo, como novedad, para el curso escolar 2017/2018 incorporaron un proyecto sobre RE realizándose en todos los niveles educativos desde Educación Infantil hasta $6^{\circ}$ de Educación Primaria. Para ello, han decidido contar con el proyecto de RE que ha realizado la editorial de libros Edelvives (Next Robótica Edelvives).

Para el nivel de $6^{\circ}$ de Primaria, nivel investigado en este estudio, se han seleccionado los paquetes de Lego WeDo 2.0, es decir, paquetes de Lego para construir un robot y programarlo con el software WeDo 2.0, además de contener un manual de montaje de todas las posibilidades y diferentes robots que se pueden construir con cada paquete de los comentados.

Todo ello, va acompañado de un manual didáctico redactado por la editorial donde propone diferentes proyectos para realizar a lo largo de todo el año, con su parte didáctica realizada a través de una metodología basada en la cooperación entre los alumnos.

Este proyecto de RE se lleva a cabo en una de las dos horas semanales que el centro escolar posee para elegir, de forma autónoma, el contenido de estas.

El proyecto ha durado todo el curso escolar y se ha dividido en tres subproyectos, uno por trimestre. El primer subproyecto ha sido la creación de oso perezoso, el cual emite sonidos cuando se dan unas circunstancias, aparte de realizar diferentes movimientos. Con este oso perezoso se trabajan también diferentes contenidos relacionados con los animales vertebrados. El segundo proyecto ha sido la creación de una canasta con una catapulta que lanza el balón, la cual emite un sonido de palmas cuando el balón entra dentro de la canasta y con la que, además, se trabajan las máquinas simples, la energía y la materia y las fuerzas. Para el tercer trimestre se ha creado un sapo corredor, con el que se trabajan los animales vertebrados y los ecosistemas.

El docente ha decidido realizar los proyectos en pareja para la parte de exploración, creación e investigación, siendo en grupos de 4 personas para las fases de compartir la experiencia a través de una exposición visual y explicativa de lo realizado, teniendo como apoyo una presentación, realizada por los propios alumnos, y la demostración de los movimientos del robot. Esta parte se tuvo que hacer con más detenimiento, sobre todo en el primer proyecto, para mejorar las exposiciones y enseñar a los alumnos a desenvolverse mejor en público.

\subsection{Instrumentos de recogida de información}

Para realizar esta investigación hemos realizado un cuestionario con preguntas cerradas, dirigido al alumnado, y una entrevista semiestructurada, dirigida al docente. El motivo es poder contrastar la percepción que poseen los estudiantes en relación con el proyecto de robótica realizado, su motivación y los aspectos que influyen en esta, así como en relación con la cooperación que han llevado a cabo durante el desarrollo del proyecto, así como lo que percibe el docente ante estos mismos aspectos.

El cuestionario (anexo I) consta de 59 preguntas con respuesta cerrada de única elección con diferentes opciones (muy en desacuerdo; en desacuerdo; ni de acuerdo ni en desacuerdo; de acuerdo; o muy de acuerdo), lo cual nos va a servir para analizar el nivel de intensidad que perciben ante una afirmación o negación 
presente en la pregunta. Está dividido en tres secciones: las preguntas de las secciones 1 (preguntas de la 1 a la 19) y 2 (preguntas de la 20 a la 45), hacen referencia a la evaluación de los deseos básicos, basadas en las preguntas que Jiménez (2017) propone en su libro para evaluar los deseos, adaptadas a las necesidades y las características de esta investigación (tabla 1).

TABLA 1. Secciones 1 y 2 del cuestionario: deseos e ítems

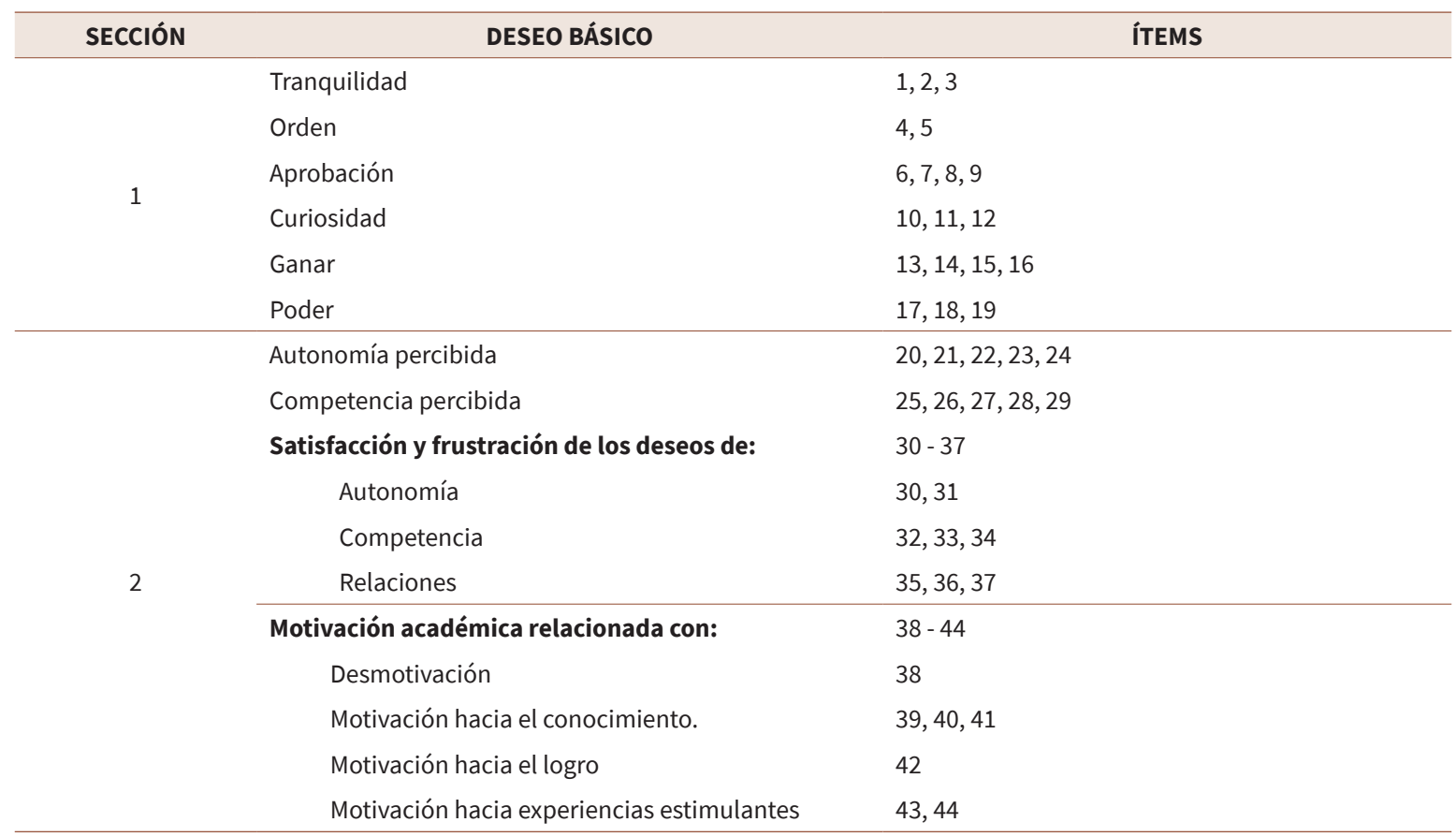

La sección 3 (preguntas de la 45 a la 59), trata sobre el trabajo cooperativo que han realizado durante el desarrollo del proyecto, donde hemos tenido en cuenta las características de nuestra investigación, así como la de los alumnos para realizarlo.

La entrevista (anexo II), por su lado, consta de 8 preguntas abiertas a contestar por el docente de forma justificada, teniendo algunas de ellas más de una cuestión por resolver. Además, al ser semiestructurada, pueden surgir preguntas que se realicen en el momento u otras que no se lleguen a plantear, en función de las respuestas que previamente vaya proporcionando la persona entrevistada.

Las preguntas que se han realizado en esta entrevista han sido obtenidas a partir de lo considerado en el cuestionario, con la finalidad de que haya una relación directa entre ambos para realizar una triangulación sobre la motivación que le produce la RE a los estudiantes, así como el trabajo colaborativo que han realizado durante el transcurso de este proyecto, visto tanto desde el punto de vista del alumnado como del docente.

\section{RESULTADOS}

En primer lugar, mostraremos los principales resultados obtenidos del cuestionario. De todos los deseos (gráfico 1) que influyen en que un estudiante esté más o menos motivado hacia un hacer, específicamente en la RE, es el orden el que prevalece sobre los demás, con una media de intensidad de un 4 sobre 5 entre los encuestados, dándole una gran importancia a las rutinas y hábitos que este proyecto precisa para su 
GRÁFICO 1. Puntuaciones medias de la sección 1 del cuestionario

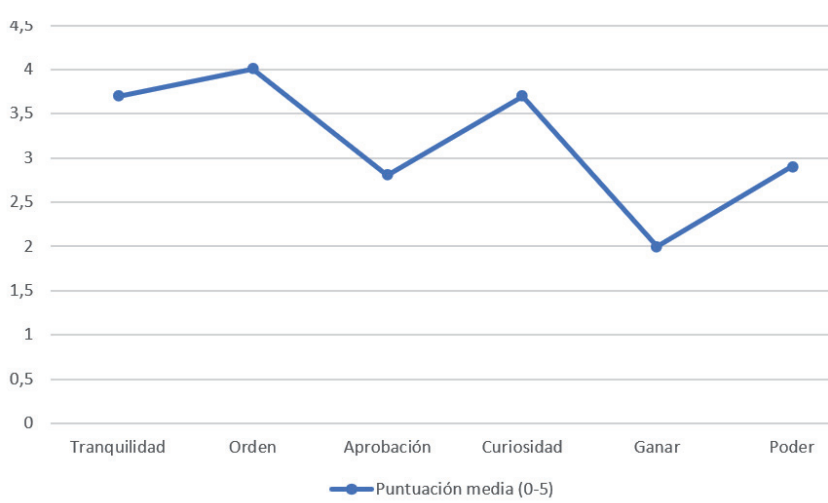

GRÁFICO 2. Puntuaciones medias sección 2 del cuestionario

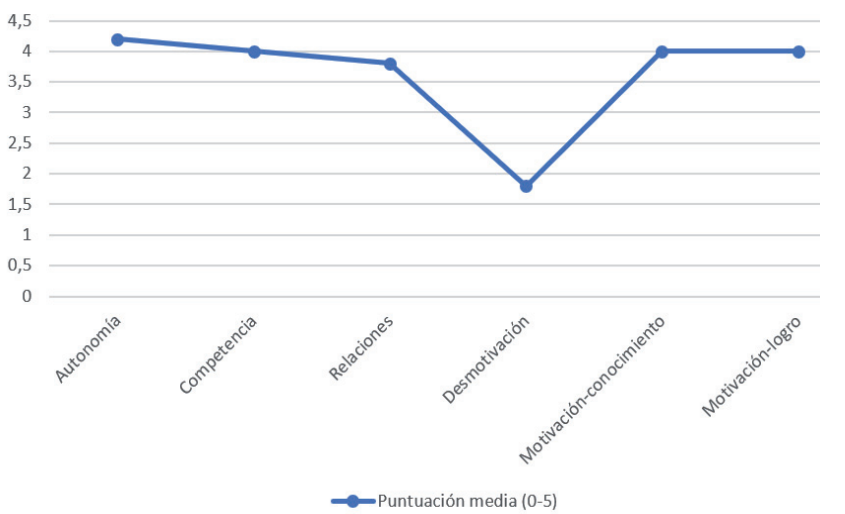

buen desarrollo. Este deseo es seguido por el de la curiosidad, con un 3,7 sobre 5 .

Ganar es el deseo menos influyente en la motivación hacia este proyecto, con un 2 sobre 5 . Para los estudiantes no es un requisito indispensable. El deseo de aprobación de los compañeros es un deseo de influencia moderada (2,8 sobre 5 ) junto con el de poder (2,9 sobre 5).

En cuanto a la segunda sección del cuestionario (gráfico 2), cabe resaltar que, por lo general, este grupo de trabajo posee buena satisfacción en cuanto a las relaciones que poseen con sus compañeros, con un 3,8 sobre 5 , siendo un grupo de trabajo poco conflictivo, en el que no destacan las peleas o discusiones. Sienten también buena satisfacción con sus capacidades y habilidades que consideran que poseen para resolver los retos planteados en el proyecto. Lo que significa que poseen buena satisfacción hacia la competencia y autonomía que perciben de ellos mismos, con un 4 y un 4,2 sobre 5 respectivamente.

Para finalizar, destacamos la gran motivación académica que muestran, pues el grado de desmotivación es mínimo con un 1,8 sobre 5. La motivación intrínseca relacionada con el conocimiento y en relación con el logro, es muy buena, superando el 4 en ambos casos. Queda patente que este proyecto de robótica ha despertado una gran curiosidad y una motivación intrínseca más que suficiente.

En cuanto a los resultados más destacados (gráfico 3) de la sección 3 (trabajo cooperativo) de este cuestionario, nos hemos encontrado con un porcentaje muy elevado de estudiantes que consideran que trabajar de forma cooperativa es beneficioso. Además de aprender nuevos conocimientos, consideran que se aprende más en equipo, logrando resultados de calidad. Suelen resolver los conflictos con paciencia y de forma pacífica. Aportan ideas de manera individual, apoyan las sugerencias de los compañeros de grupo y ayudan a otros cuando surgen dificultades. Afirman que, en la mayoría de las ocasiones, todos los miembros colaboran en la realización exitosa de los proyectos.

La gran mayoría de estos estudiantes se sienten bien trabajando a través de esta metodología y le gusta cooperar con sus compañeros para conseguir resolver los retos planteados. No encontramos ningún caso que esté en desacuerdo con la anterior afirmación, lo que nos hace entender que las sensaciones y emociones que se sienten, al trabajar así, son positivas y beneficiosas para ellos, por lo que prefieren trabajar de forma cooperativa a trabajar con una metodología más individualizada.

En la entrevista, el docente ha corroborado todos estos datos comentados, además de contarnos experiencias surgidas en el aula. Se ha quedado sorprendido al ver el resultado que ha obtenido al realizar este 
GRÁFICO 3. Resultados expresados en frecuencias de la sección 3 del cuestionario

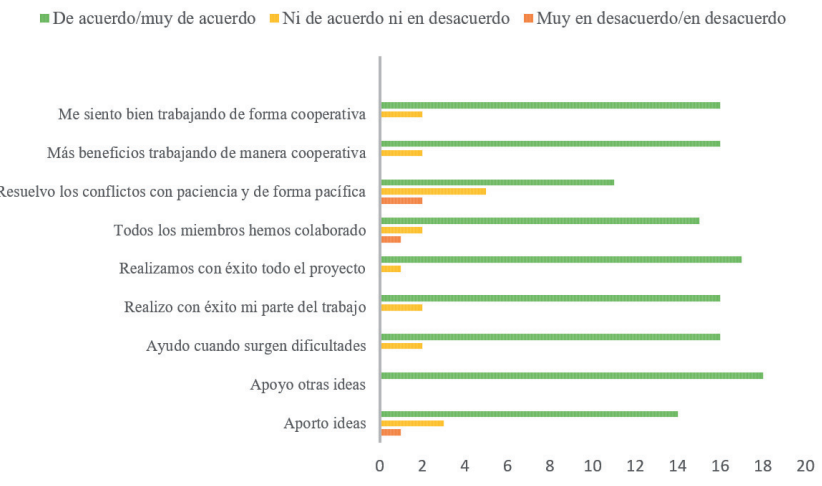

proyecto, pues la motivación del alumnado ha sido impresionante, estaban deseando que llegase la hora de robótica, comentaba, hasta el punto de que los propios estudiantes pedían nuevos y más complejos retos conforme iban avanzando las sesiones e iban cogiendo confianza con la metodología y los materiales. También ha destacado el gran compañerismo que ha visto en el aula y la ayuda que se han prestado los unos a los otros cuando lo necesitaban, así como la responsabilidad que ha observado en el cuidado del material: al ser partícipes de la

actividad, tenían cierta responsabilidad en el material y lo cuidaban por el simple hecho de que si se perdía alguna pieza ellos eran los responsables.

El entrevistado recomienda la utilización de estos dos recursos educativos por su experiencia positiva, pues, como comenta, se pueden enlazar muchas cosas a raíz de la robótica, se pueden trabajar de forma interdisciplinar muchas áreas, siempre y cuando se adapta a las necesidades del alumnado y del contexto educativo.

\section{CONCLUSIONES Y DISCUSIÓN}

La finalidad de este estudio fue analizar el impacto que tiene la RE en la motivación y el trabajo cooperativo entre estudiantes de Educación Primaria de un centro educativo de la Región de Murcia. Teniendo en cuenta los resultados obtenidos concluimos que:

- El proyecto de robótica suscita en los estudiantes un aumento del interés y la motivación, pues les hace responsables de sus materiales, así como partícipes de su aprendizaje, siendo unos sujetos activos en este.

- La metodología de trabajo cooperativo ha provocado en este grupo de alumnos una diversión mientras aprenden, lo que ha propiciado llegar a conocimientos más amplios y complejos, así como a la resolución de retos a partir de la ayuda y el aprendizaje entre iguales. Observamos pues, los beneficios de este trabajo cooperativo dentro del proyecto de robótica.

Coincidiendo con Ruiz (2007), con esta investigación consideramos que, con una adecuada planificación, la RE puede ser de utilidad para generar entornos de aprendizaje con tecnologías que constituyan un cambio significativo en el aprendizaje y las relaciones. Esto es posible gracias a su interdisciplinariedad y al desarrollo de habilidades como el trabajo en equipo. Es necesario seguir una perspectiva holística del aprendizaje, donde haya una interacción entre iguales y un trabajo cooperativo para crear su propio aprendizaje, buscando soluciones para resolver conflictos y retos en común.

Las emociones son un aspecto muy importante dentro del proceso de enseñanza-aprendizaje, pues un estudiante necesita en gran medida emocionarse para poder aprender. Este proceso le debe provocar una emoción que le produzca sentir un interés hacia el proceso en el que está implicado, con el fin de que los 
conocimientos sean significativos. Esto lo hemos visto patente en los resultados del cuestionario cuando los alumnos nos contaban sus intereses y los deseos básicos que los movían en este proyecto. Tampoco puede decirse que estos resultados sean inesperados, ya que hay una evidencia empírica acumulada que afirma que a partir de la emoción y la motivación se produce el aprendizaje, como destacan Barrera (2015), Cabero et al. (2017), García y Reyes (2012), López y Yuste (2017), Merino et al. (2017) o Moreno et al. (2012).

Los resultados obtenidos confirman que la RE y el trabajo cooperativo tienen una relación beneficiosa y efectiva y están en la línea de las conclusiones obtenidas por varios autores (Alimisis, 2013; Caballero y García-Valcárcel, 2020; García y Reyes, 2012; Souza et al., 2018) que resaltan dicha relación y las ventajas que proporciona, así como la creación de técnicas cooperativas que se han realizado durante este proyecto y que han servido para crear aprendizajes significativos, como fundamentan Acosta et al. (2015). Por lo tanto, la RE puede ser de utilidad más allá del aprendizaje de contenidos relacionados con asignaturas STEM (Jung y Won, 2018; Yang et al., 2020).

En futuros cursos se podría incluir la RE en diferentes áreas curriculares para conocer su influencia en el proceso de aprendizaje de los estudiantes, analizando otro tipo de variables sin llegar a aislar la motivación y el trabajo cooperativo (aspectos centrales en este estudio) y no solamente trabajando la robótica de manera específica, sino también de manera transversal con el apoyo de otros docentes. También será de interés seguir analizando el avance de este proyecto en futuros cursos académicos para poder comprobar su evolución en la motivación y el trabajo cooperativo de los estudiantes.

\section{REFERENCIAS}

Acosta, M. Forigua, C. P., y Navas, M. A. (2015). Robótica educativa: un entorno tecnológico de aprendizaje que contribuye al desarrollo de habilidades (Trabajo Fin de Maestría). Pontificia Universidad Javeriana, Bogotá, Colombia.

Alimisis, D. (2013). Educational robotics: Open questions and new challenges. Themes in Science and Technology Education, 6(1), 63-71.

Ayuso, M. A. (2016). Robótica educativa: una nueva metodología activa para fomentar la motivación, la creatividad y el aprendizaje significativo en la etapa de primaria (Trabajo Fin de Grado). Universidad de Valladolid, Valladolid, España.

Barrera, N. (2015). Uso de la robótica educativa como estrategia didáctica en el aula. Praxis \& Saber. Revista de Investigación y Pedagogía, 6(11), 215-234.

BMaker (s/f). bMaker. Solución integral para aprender robótica y programación en el aula. Recuperado de: https://bmaker.es/

Caballero, Y., y García-Valcárcel, A. (2020). Fortaleciendo el pensamiento computacional y habilidades sociales mediante actividades de aprendizaje con robótica educativa en niveles escolares iniciales. Píxel-Bit. Revista de medios y educación, (58), 117-142. https://doi.org/10.12795/pixelbit.75059

Cabero, J., Fernández, B., y Marín, V. (2017). Dispositivos móviles y realidad aumentada en el aprendizaje del alumnado universitario. RIED. Revista Iberoamericana de Educación a Distancia, 20(2), 167-185. http://dx.doi.org/10.5944/ried.20.2.17245 Cabrera, J. M. (2015). Computer Programming and Robotics in Basic. Avances en Supervisión Educativa. Revista de la Asociación de Inspectores de Educación de España, (24), 1-26.

Cervera, E., y Casañ, G. A. (2015). Robot Programming Network: un sistema distribuido para el aprendizaje de la programación de robots. ReVisión, 8(1), 63-72.

Cubides, H. Cuvi, L. Cuzco, J., y Ordoñez E. (2012). Diseño, construcción e implementación de una plataforma robótica multifuncional con propósitos didácticos DINGO 1.0. INGENIUS, (7), 29-34. https://doi.org/10.17163/ings.n7.2012.04

Fagin, B., y Merkle, L. (2003). Measuring the effectiveness of robotics in teaching computer science. ACM SIGCSE Bulletin, 35(1), 307-311. http://doi.org/10.1145/792548.611994 
Fernández, A. (2006). Metodologías activas para la formación de competencias. Educatio siglo XXI, 24, 35-56.

García, Y., y Reyes, D. (2012). Robótica educativa y su potencial mediador en el desarrollo de las competencias asociadas a la alfabetización científica. Revista Educación y Tecnología, (2), 42-55.

González, A., y Redondo, A. (2013). AIToy 1, un robot neo-educativo con emociones. Informática Educativa Comunicaciones, (18), 51-62.

Heredero, E. S., y Oliva, A. (2014). Experiencias y recursos con las tics para la atención al alumnado con necesidades educativas especiales. Acta Scientiarum. Education. 36(2), 279-286.

Hernández, R., Fernández, C., y Baptista, P. (2010). Metodología de la investigación. Mc Graw Hill.

Jiménez, L. (2017). El poder y la ciencia de la motivación. Cómo cambiar tu vida y vivir mejor gracias a la ciencia de la motivación. Amazon.

Jófili,Z. (2002). Piaget, Vygotsky, Freire e a Construção do Conhecimento na Escola. Educação: Teorias e Práticas, 2 (2), 191-208.

Johnson, D. W., Johnson, R., y Holubec, E. (2004). El aprendizaje cooperativo en el aula. Paidos.

Jung, S., y Won, E. (2018). Systematic Review of Research Trends in Robotics Education for Young Children. Sustainability, 10(4), 905. https://doi.org/10.3390/su10040905

Latorre, A., Rincón D., y Arnal, J. (2005): Bases Metodológicas de la Investigación Educativa. Ediciones experiencia.

López, V., y Yuste, R. (2017). EMOROBOTIC: Gestión Emocional a través de la Programación en Robots en Educación Primaria. En S. Pérez, G. Castellano, y A. Pina (Coords.), Propuestas de Innovación Educativa en la Sociedad de la Información (pp. 82-91). Adaya Press.

McDougall, W. (1908). An introduction to social psychology. Methuen \& Co

Merino, J. M., Villena, R., González J. A., y Cózar, R. (2017). Análisis del efecto de la robótica en la motivación de estudiantes de tercero de Educación Primaria durante la resolución de tareas de interpretación de planos. Revista de Estudios y Experiencias en Educación, (3), 163-173.

Morales, P (2017). La robótica educativa: una oportunidad para la cooperación en las aulas. En J. Ruiz, J., J. Sánchez, y E. Sánchez (Eds.). Innovación docente y uso de las TIC en educación. UMA Editorial.

Moreno, I., Muñoz, L., Serracín, J. R., Quintero, J., Pittí, K., y Quiel, J. (2012). La robótica educativa, una herramienta para la enseñanza-aprendizaje de las ciencias y las tecnologías. Revista Teoría de la Educación: Educación y Cultura en la Sociedad de la Información, 13(2), 74-90.

Ruiz, E. (2007). Educatrónica: innovación en el aprendizaje de las ciencias y la tecnología. Ediciones Díaz de Santos.

Sánchez, L., Rodríguez, J., y Narváez, R. (2005). Hacia un laboratorio escolar de robótica remoto. http://sedici.unlp.edu. ar/bitstream/handle/10915/22863/Documento_completo. pdf?sequence $=1$

Souza, I. M. L., Andrade, W. L., Sampaio, L. M. R., y Araujo, A. (2018). A Systematic Review on the use of LEGO ${ }^{\circledR}$ Robotics in Education. 2018 IEEE Frontiers in Education Conference (FIE), 1-9. https://doi.org/10.1109/FIE.2018.8658751

Stake, R. E. (2005). Investigación con estudio de casos. Morata.

Trujillo, J. (1998, octubre 20-23). Trabajo en equipo, una propuesta para los procesos de enseñanza - aprendizaje [Comunicación en congreso]. IV Congresso RIBIE, Brasilia, Brasil.

Vallejo, R., y De Franco, M. F. (2009). La triangulación como procedimiento de análisis para investigaciones educativas. REDHECS, 7(4). 117-133.

Vigueras, J. V., y Villalba, K. O. (2017). Education and Educative Robotics. Revista de Educación a Distancia, 54(11), 1-13. https://doi.org/10.6018/red/54/11

Yang, K., Liu, X., y Chen, G. (2020). The Influence of Robots on Students Computational Thinking: A Literature Review. International Journal of Information and Education Technology, 10(8), 5. https://doi.org/10.18178/ijiet.2020.10.8.1435 


\section{ANEXOS}

Anexo I: cuestionario realizado a los alumnos. https://goo.gl/q1xsbE

Anexo II: entrevista semiestructurada personalizada para el docente.

1. La motivación que lleva a una persona a que se interese más por unas cosas que por otras está influenciada por los deseos básicos que posee esa persona. Dentro de estos deseos, encontramos el ejercicio, la tranquilidad, el orden, la aprobación, la curiosidad, el ganar o el poder. ¿Cuáles de estos deseos has podido observar en los alumnos con más fuerza y cuáles con menos? Pon algún ejemplo.

2. La autonomía, entendida como la percepción que se tiene sobre la capacidad para decidir y la posibilidad de gestionar una situación, también entra en juego en la motivación que mueve a una persona a realizar un acto con más interés o menos. ¿Qué dificultades más notables has encontrado durante el desarrollo de este proyecto?

3. La competencia entendida como las capacidades y habilidades necesarias para conseguir un grado de efectividad, suficiencia o éxito, es otro factor de los que influyen en la motivación de una persona. ¿Cómo consideras que afecta esta competencia a los niños en relación al proyecto que han realizado sobre robótica en el aula? (¿evolución a lo largo del proyecto? ¿Han ido adquiriendo esas competencias poco a poco?) ¿Y este proyecto a la competencia del alumnado? (¿Los has visto capacitados tanto cognitivamente como madurativamente para realizar este tipo de tareas?)

4. ¿En qué parte del proyecto has encontrado más frustración de estos deseos y necesidades básicas que fomentan y engloban la motivación que siente una persona al realizar una tarea? Pon algún ejemplo.

5. ¿Has sentido que este proyecto ha conllevado una desmotivación en el alumnado o, por el contrario, fomenta la motivación en el niño o niña de tal forma que le afecta incluso en el rendimiento en otras áreas de trabajo en el aula?

6. Vamos a hablar ahora sobre la cooperación en la que han participado los alumnos durante el desarrollo de este proyecto de robótica educativa. ¿Es la primera vez que trabajan de forma cooperativa? ¿Por qué decides trabajar de forma cooperativa en robótica educativa? ¿Qué dificultades observaste en los alumnos a la hora de trabajar con esta metodología?

7. ¿Qué rutinas o pautas han seguido los alumnos para trabajar con esta metodología?

8. Para finalizar, ¿qué beneficios consideras que ha aportado esta metodología de trabajo cooperativo en relación al proyecto en el que se ha llevado a cabo? 